\title{
On the Augmented Lagrangian Dual for Integer Programming
}

\author{
N.L. Boland and A. C. Eberhard*
}

\begin{abstract}
We consider the augmented Lagrangian dual for integer programming, and provide a primal characterization of the resulting bound. As a corollary, we obtain proof that the augmented Lagrangian is a strong dual for integer programming. We are able to show that the penalty parameter applied to the augmented Lagrangian term may be placed at a fixed, large value and still obtain strong duality for pure integer programs.
\end{abstract}

\section{Introduction}

Duality has long been a powerful tool in Integer Programming (IP). Several dual problems have been proposed for IP (see, e.g. Nemhauser and Wolsey [34] Chapter II.3), including the Lagrangian dual [17], the surrogate dual [18], the subadditive dual [20, 23, 24], and more recently, the inference dual [22]. The Lagrangian dual has given rise to a wealth of algorithms, both as a heuristic and through the provision of bounds for use in exact methods such as branch-and-bound. Surrogate duality has been less well explored, but methods have been developed for solving surrogate duals, and for embedding them in methods for solving the primal IP, notably those of Karwan and Rardin [25, 26]. The value of the inference and subadditive duals appears to have been primarily for their use in sensitivity analysis and the study of the value function for IP [3,40]. One surprising new development is the application of Z-transforms and complex variable techniques by Lasserre [28, 29] to study the value function. Very recently, algorithms for solving the subadditive dual for both linear and nonlinear IP have been developed [27, 31].

One reason for the prevalence of Lagrangian duality has been its tractability. However this advantage has a concomitant downside: it does not, in general, provide a strong bound. As noted in [26], the surrogate dual bound cannot be worse than the Lagrangian dual bound obtained through relaxation of the same set of constraints, and can be better. This is verified in other studies [21, 19, 16]. Strong duality in a form that allows practical, computationally useful methods to estimate bounds for IP has been elusive, although the recent work of Klabjan [27] and Li et al. [31] offers great promise.

By contrast, for nonlinear, nonsmooth optimisation there have been a number of strong duality schemes proposed in the last 10 years. The augmented Lagrangian dual has been of particular interest in this area. In convex optimisation, algorithms to solve the augmented Lagrangian dual were found to be more robust, and converged under less stringent assumptions, than their standard Lagrangian dual predecessors [36]. Early application of the augmented Lagrangian in IP [38] was aimed at producing better dual bounds at the root node of the branch-and-bound tree. Li and Sun [30] report some more recent progress with strong duality schemes. In other lines of research, the potential for their application has been observed but not extensively studied. Theory cast in a very general framework may be found in the work of Burachik and Rubinov [6,7], which requires some standard assumptions for unstructured nonlinear problems that are less natural for IPs. The approach of [6] and [7] provides justification for the penalty methods applied to small nonlinear IPs in [8].

One of the challenges for applying the augmented dual in IP computational methods is that the augmented Lagrangian term destroys the natural separability properties of the Lagrangian dual. However this can be overcome by the use of an approach known as the alternating direction method of multipliers $[5,14]$, which is enjoying recent attention in the literature. Thus the augmented Lagrangian dual for IP warrants further attention.

In this paper, we contribute to the theory of the augmented Lagrangian dual for IP so as to provide insight into how it obtains better bounds than the standard Lagrangian dual. Our main result is a

\footnotetext{
*Email addresses of the authors: Natashia.Boland@newcastle.edu.au, andy.eb@rmit.edu.au This research was supported by the ARC Discovery grant no. DP140100985.
} 
primal characterization of the augmented Lagrangian dual value which in some sense mirrors that given in [34] Chapter II.3.6 as Theorem 6.2 (originally proved in [17]) for the standard Lagrangian dual. The primal description of the standard Lagrangian dual is maximization of the original objective over the intersection of the set of points satisfying the relaxed constraints and the convex hull of points satisfying the constraints that are not relaxed, including integrality. In other words, the action of the standard Lagrangian dual is to convexify the integer points feasible for the constraints that are not relaxed. (This is expressed more precisely in equation (1) below.) We show that the augmented Lagrangian dual convexifies only a subset of these points, convexifying the integer points that satisfy the constraints not relaxed and that do not violate the constraints that are relaxed by too much, within a parameterized framework controlling a measure of the degree of violation of the relaxed constraints. The fact that the augmented Lagrangian term should act to control the violation of the relaxed constraints is intuitively clear: here we provide a rigorous formulation showing how that occurs.

A motivation for considering this direction of research arises from its links to standard Lagrangian methods and their role in IP. For many well-known applications, such as airline crew scheduling and cutting stock problems, the standard Lagrangian dual yields a bound with very small duality gap, and is solved in its primal form via the technique of column generation - undoubtedly one of the success stories of integer programming [13]. Since Theorem 6.2 (Chapter II.3.6, [34]) underpins the use of column generation as a technique for solving the standard Lagrangian dual, it is our hope that providing an analogous result for the augmented Lagrangian dual will stimulate new methods for the latter. Furthermore, column generation is an active subject of current research, with new ideas such as stabilization and dynamic constraint aggregation [32, 15, 2] emerging in recent years, aimed at accelerating convergence. With the augmented Lagrangian providing greater stability and better convergence properties than standard Lagrangian approaches in convex optimization, the idea that the augmented Lagrangian could also lead to convergence improvements in IP is an intriguing possibility.

As a corollary of our primal characterization, we are able to derive strong duality for the augmented Lagrangian dual. In the case of pure integer programs with bounded domains, we also present a stronger result, showing that the zero duality gap is attained for finite values of the augmented penalty term parameter. We demonstrate that strong duality for the augmented Lagrangian dual for IP can also be deduced from the results of [6], but the derivation is somewhat indirect, and does not yield any of the other results we give here.

\section{Lagrangian and Augmented Lagrangian Duals}

The standard Lagrangian dual in integer programming is obtained as follows. Let the mixed integer program (IP) be defined by $z^{I P}=\sup \{c x \mid A x=b, x \in X\}$, with $A$ an $m \times n(m \leq n)$ of full rank and $X=\left\{x \geq 0 \mid D x=d, x_{i} \in \mathbf{Z} \forall i \in J\right\}$, where $D$ is $\tilde{m} \times n$ and $J \subseteq\{1, \ldots, n\}$. Usually the (IP) is taken to be structured so that $X$ includes integrality constraints, simple bounds on variable, and other simple constraints. We assume $X$ is closed, and that the value $z^{I P}$ and its linear programming relaxation $z^{L P}$ are finite. Form the Lagrangian relaxation

$$
z^{L R}(\lambda):=\sup _{x \in X}[c x+\lambda(b-A x)]
$$

and the associated Lagrangian dual $z^{L D}:=\inf _{\lambda} z^{L R}(\lambda)$. Clearly $z^{L R}(\lambda)$ provides an upper bound on (IP), $\left(z^{L R}(\lambda) \geq z^{I P}\right.$ for all $\left.\lambda\right)$, and $z^{L D}$ provides the best such bound and $z^{L D} \leq z^{L P}$.

The Lagrangian dual has an elegant primal characterization [17, 34]:

$$
z^{L D}=\sup \{c x \mid A x=b, x \in \operatorname{conv}(X)\},
$$

where $\operatorname{conv}(C)$ denotes the smallest convex set containing $C$. So if $X$ does not have the integrality property $^{1}$, the bound from the Lagrangian dual may be better than that obtained from the LP relaxation of (IP).

Let us consider the augmented Lagrangian relaxation

$$
z_{\sigma}^{L R+}(\lambda):=\sup _{x \in X}[c x+\lambda(b-A x)-\sigma \rho(b-A x)],
$$

\footnotetext{
${ }^{1}$ The set $X$ satisfies the "integrality property" if ignoring the integrality constraints in the definition of $X$ yields $\operatorname{conv}(X)$.
} 
where $\rho: \mathbf{R}^{m} \rightarrow \mathbf{R}$ is a penalty term such as a polyhedral norm or the square of the standard 2-norm and $\sigma \geq 0$ is treated as a fixed parameter.

Assumption 1: $\rho$ is of the form $\rho(x)=\psi(\|x\|)$ for some norm $\|\cdot\|$ on $\mathbf{R}^{m}$, where $\psi: \mathbf{R}_{+} \rightarrow \mathbf{R}_{+}$is a convex, monotonic increasing function for which $\psi(0)=0$ and there exists $\delta>0$ for which

$$
\liminf _{a \rightarrow+\infty} \frac{\psi(a)}{a} \geq \delta>0
$$

with $\operatorname{diam}\{a \mid \psi(a) \leq \delta\} \downarrow 0$ as $\delta \downarrow 0$.

An advantage of using this form of penalty arises from the equivalence of norms in finite dimensions. Recall all norms $\|\cdot\|$ in finite dimensions have $0<\gamma<1$ such that $\frac{1}{\gamma}\|y\| \geq\|y\|_{\infty} \geq \gamma\|y\|$ for all $y$. Thus $\tilde{\psi}(\|x\|):=\psi\left(\frac{1}{\gamma}\|x\|\right) \geq \psi\left(\|x\|_{\infty}\right) \geq \psi(\gamma\|x\|):=\hat{\psi}(\|x\|)$ where both $\tilde{\psi}$ and $\hat{\psi}$ satisfy Assumption 1 . Thus if we can prove strong duality for any one norm we have it for all norms.

Clearly $z_{\sigma}^{L R+}(\lambda) \leq z^{L R}(\lambda)$ for $\sigma \geq 0$. It is obvious that for any $\sigma \geq 0, z_{\sigma}^{L R+}(\lambda)$ provides an upper bound on $z^{I P}$ for any $\lambda$, and $z_{\sigma}^{L D+}:=\inf _{\lambda} z_{\sigma}^{L R+}(\lambda) \leq z^{L D}$ is the best such bound. In Theorem 1 below, we provide a primal expression for $z_{\sigma}^{L D+}$ which provides insight into how the augmented Lagrangian dual can yield a strictly better bound than that of the standard Lagrangian. As we shall subsequently see, as $\sigma$ increases, the former bound becomes exact.

Denote the recession directions of a convex set $K \subseteq \mathbf{R}^{n}$ by $0^{+} K$ (the directions in which the convex set $K$ is unbounded ${ }^{2}$ ). Denote the linear programming relaxation of the constraint set $X$ by $X^{L P}$. This latter set is a polyhedral set and so we may denote by ext $X^{L P}$ the finite set of extremal points of the set $X^{L P} \cap\left(\operatorname{lin} X^{L P}\right)^{\perp}$ obtained by factoring out its lineality space $\operatorname{lin} X^{L P}=0^{+} X^{L P} \cap\left(-0^{+} X^{L P}\right)$. Thus $0^{+} X^{L P}=0^{+} X^{L P} \cap\left(\operatorname{lin} X^{L P}\right)^{\perp}+\operatorname{lin} X^{L P}$ (see [35, page 65]), where $0^{+} X^{L P} \cap\left(\operatorname{lin} X^{L P}\right)^{\perp}$ is a pointed cone ${ }^{3}$. Hence a Minkowski decomposition exists of the form $X^{L P}=\operatorname{conv}\left(\operatorname{ext} X^{L P}\right)+0^{+} X^{L P}$ where $0^{+} X^{L P}$ may contain a lineality space. As we assume $z^{L P}<+\infty$ we have $c v \leq 0$ for all $v \in V:=0^{+} X^{L P} \cap \operatorname{ker} A$. Moreover for all $v \in \operatorname{lin} X^{L P} \cap \operatorname{ker} A$ we must have $c v=0$ for $z^{L P}<+\infty$. We can derive our duality results under three alternative assumptions on the data of the MIP. First we can assume the LP relaxation of the MIP has a bounded solution set; we use the weaker assumption that optimal solution set of the LP relaxation does not contain a lineality space. This requires the condition that $\left(\operatorname{ker} A \cap \operatorname{lin} X^{L P}\right) \cap c^{\perp}=\{0\}$, where $c^{\perp}=\{x \mid c x=0\}$. If in addition $z^{L P}<+\infty$ (a standing assumption for this paper) then $\operatorname{lin} X^{L P} \cap \operatorname{ker} A=\{0\}$ and $0^{+} X^{L P} \cap \operatorname{ker} A$ is a pointed cone (if $v,-v \in \operatorname{lin} X^{L P} \cap \operatorname{ker} A$ then $c v=0$ if $z^{L P}<+\infty$ so $v=0$ under our assumption). Second is a standard sufficient condition used in MIP to ensure a solution exists, combined with the restriction to a polyhedral norm. Third is a boundedness assumption on $X$.

Assumption 2: At least one of the following conditions hold. 1) The solution set of the LP relaxation does not contain a lineality space. 2) The matrices $A$ and $D$ have rational entries and the norm $\|\cdot\|$ used in the definition of the Lagrangian relaxation (i.e. in $\rho$ satisfying Assumption 1 ) is the $l^{\infty}$ norm. 3) $\operatorname{conv}(X)$ is bounded.

Denote the projection of $x$ onto the convex cone $V$ by $P_{V} x$. For a convex cone $V$ denote its polar $V^{\circ}:=\{y \mid y x \leq 0$, for all $x \in V\}$. Throughout the following argument we will use $V:=0^{+} X^{L P} \cap \operatorname{ker} A$, $K(\kappa):=\operatorname{conv}(X \cap\{y \mid\|b-A y\| \leq \kappa\})$ and $C(\kappa):=\left\{P_{V^{\circ}} x \mid x \in K(\kappa)\right\}$.

The following is well known and provide the orthongonal decomposition in lemma 1 below.

Proposition 1 Let $B$ be a closed convex cone. We may decompose $x$ into an orthogonal sum $x=$ $x_{1}+x_{2}$ where $x_{1}:=P_{B} x \in B$ and $x_{2}:=P_{B^{\circ}} x \in B^{\circ}$.

Lemma 1 Posit Assumption 2. Then we have an orthogonal decomposition $x=x_{1}+x_{2}$ for $x_{1}:=$ $P_{V} x \in V$ and $x_{2}:=P_{V} \circ \in V^{\circ}$ with $\kappa \mapsto C(\kappa)$, convex valued and uniformly bounded for all $\bar{\kappa} \geq \kappa \geq 0$. Hence $\kappa \mapsto \overline{C(\kappa)}$ is upper semi-continuous.

Proof. This proof may be found in the Appendix.

We will need to use the minimax theorem of Sion [39].

Proposition 2 ([39]) Let $G, H$ be convex subsets of $\mathbf{R}^{n}$ with $H$ closed and bounded. Let $f: G \times H \rightarrow$ $\mathbf{R}$ with $f(g, \cdot)$ upper semi-continuous and quasi-concave ${ }^{4}$ on $H$ for every $g \in G$. If $f(\cdot, h)$ is lower

\footnotetext{
${ }^{2}$ We have $x \in 0^{+} K$ iff there exists $\lambda_{q} \downarrow 0$ and $x^{q} \in K$ with $\lambda_{q} x^{q} \rightarrow x$

${ }^{3} \mathrm{~A}$ cone $K$ is pointed iff $K \cap(-K)=\{0\}$

${ }^{4} \mathrm{~A}$ function $x \mapsto f(x)$ is quasi-convex if $\{x \mid f(x) \leq \alpha\}$ is convex for all $\alpha$. A function $x \mapsto f(x)$ is quasi-concave if $-f$ is quasi-convex.
} 
semi-continuous and quasi-convex on $G$ for every $h \in H$, then

$$
\inf _{g \in G} \max _{h \in H} f(g, h)=\max _{h \in H} \inf _{g \in G} f(g, h) .
$$

Theorem 1 Suppose $-\infty<z^{I P} \leq z^{L P}<+\infty$ and Assumptions 1 and 2 hold. Then for any $\sigma>0$

$$
z_{\sigma}^{L D+}=\max _{\kappa \geq 0}[\sup \{c x \mid A x=b, x \in \operatorname{conv}(X \cap\{y \mid\|b-A y\| \leq \kappa\})\}-\sigma \psi(\kappa)] .
$$

The maximum in $\kappa \geq 0$ is attained by a finite value $\kappa(\sigma)$, where $\kappa(\sigma) \downarrow 0$ as $\sigma \uparrow \infty$.

Proof. We may write

$$
\begin{aligned}
z_{\sigma}^{L R+}(\lambda) & =\sup _{x \in X} \sup _{\kappa \geq 0}\{c x+\lambda(b-A x)-\sigma \psi(\kappa) \mid\|b-A x\| \leq \kappa\} \\
& =\sup _{\kappa \geq 0} \sup _{x \in X}\{c x+\lambda(b-A x)-\sigma \psi(\kappa) \mid\|b-A x\| \leq \kappa\} \\
& =\sup _{\kappa \geq 0}[\sup \{(c-\lambda A) x \mid x \in \operatorname{conv}(X \cap\{y:\|b-A y\| \leq \kappa\})\}-\sigma \psi(\kappa)]+\lambda b
\end{aligned}
$$

because the maximum of a linear functional over a set equals its maximum over its convex hull. Note that as $z_{\sigma}^{L D+}<+\infty$ there exist $\lambda$ for which $z_{\sigma}^{L R+}(\lambda)<+\infty$.

Let $\|\cdot\|_{2}$ denote the Euclidean norm, $\mathcal{T}(\kappa, \lambda):=\sup \{(c-\lambda A) x \mid x \in K(\kappa)\}$ where $K(\kappa)$ and $V$ are as chosen in Lemma 1. Furthermore we may decompose any $x \in K(\kappa)$ into an orthogonal sum $x=x_{1}+x_{2}$ with $x_{1} \in V^{\circ}$ and $x_{2} \in V=0^{+} X^{L P} \cap \operatorname{ker} A$. As noted earlier $c x_{2} \leq 0$ so it follows that

$$
\mathcal{T}(\kappa, \lambda) \leq \sup \left\{(c-\lambda A) P_{V^{\circ}}(x) \mid x \in K(\kappa)\right\}=\sup \{(c-\lambda A) y \mid y \in \overline{C(\kappa)}\} .
$$

where the last equality holds due to the boundedness and convexity of $C(\kappa)$.

Applying the uniform boundedness of $C(\kappa)$ over all $\kappa$ we have the bound $\sup \{\|y\| \mid y \in C(\kappa)\} \leq$ $M<+\infty$. hence the bound $\mathcal{T}(\kappa, \lambda) \leq\|c-\lambda A\|_{2} M$. Thus (5) is bounded below by $z_{\sigma}^{L D+}$ (as $\left.z_{\sigma}^{L D+}=\inf _{\lambda} z_{\sigma}^{L R+}(\lambda)\right)$ and above by the difference $\|c-\lambda A\|_{2} M+\|\lambda\|_{2}\|b\|_{2}-\sigma \psi(\kappa)$ so if $\kappa$ solves (5) we have

$$
z_{\sigma}^{L D+} \leq\|c-\lambda A\|_{2} M+\|\lambda\|_{2}\|b\|_{2}-\sigma \psi(\kappa) .
$$

Hence a contradiction follows from the coercivity of $\psi(\cdot)$ for any fixed $\sigma$ and $\lambda$ if $\kappa$ assumed to be larger than a constant $B(\lambda)$ (which is bounded for bounded $\lambda$ ). Note that $B(\lambda)$ is also $\sigma$-dependent, but it is non-increasing for increasing $\sigma$, thus under the assumption that $\sigma \geq \bar{\sigma}>0$ (any $\bar{\sigma}>0$ ) this dependence can be ignored.

Define $F(\kappa, \lambda):=\mathcal{T}(\kappa, \lambda)+\lambda b=\sup \{c x+\lambda(b-A x) \mid x \in K(\kappa)\}$. Then (as $c z \leq 0$ for all $z \in V)$

$$
-\infty<z^{I P} \leq F(\kappa, \lambda) \leq \sup \{c x+\lambda(b-A x) \mid x \in \overline{C(\kappa)}\} \leq\|c-\lambda A\|_{2} M+\|\lambda\|_{2}\|b\|_{2}<+\infty,
$$

where the upper bound is finite due to the compactness of $\overline{C(\kappa)}$. Thus $F(\kappa, \lambda)$ is finite valued and moreover we claim $\kappa \mapsto F(\kappa, \lambda)$ is upper semi-continuous. As this function is monotonically nondecreasing we only have to consider a sequence $\kappa_{p} \downarrow \bar{\kappa}$ and show that $\lim _{p} F\left(\kappa_{p}, \lambda\right) \leq F(\bar{\kappa}, \lambda)$. Let $\varepsilon_{p} \downarrow 0$ and let $x_{p} \in K\left(\kappa_{p}\right)$ achieve the supremum to within $\varepsilon_{p}$. Decompose $x_{p}$ into $x_{p}^{1} \in \bar{V}:=0^{+} X^{L P} \cap \operatorname{ker} A$ and $x_{p}^{2} \in \overline{C\left(\kappa_{p}\right)}$. Using the upper semi-continuity of $\overline{C(\cdot)}$ we may take a convergent subsequence (and after re-numbering) we have $x_{p}^{2} \rightarrow x^{2} \in \overline{C(\bar{\kappa})}$. If $\left\{x_{p}^{1}\right\}$ is also bounded we may also extract a convergent subsequence (and after re-numbering) have $x_{p}^{1} \rightarrow x^{1}$ with $x:=x^{1}+x^{2} \in K(\bar{\kappa})$ (and in this case we actually have $\left.x^{2} \in C(\bar{\kappa})\right)$. Then $\lim _{p} F\left(\kappa_{p}, \lambda\right)=c x+\lambda(b-A x) \leq F(\bar{\kappa}, \lambda)$. When $\left\{x_{p}^{1}\right\}$ is unbounded, by $(7)$ we have $F(\cdot, \lambda)$ uniformly bounded so

$$
0=\lim _{p} \frac{1}{\left\|x_{p}^{1}\right\|_{2}}\left(c x_{p}+\lambda\left(b-A x_{p}\right)\right)=\lim _{p}\left(c\left(\frac{x_{p}^{1}+x_{p}^{2}}{\left\|x_{p}^{1}\right\|_{2}}\right)+\frac{1}{\left\|x_{p}^{1}\right\|_{2}} \lambda\left(b-A x_{p}^{2}\right)\right)=\lim _{p} c\left(\frac{x_{p}^{1}}{\left\|x_{p}^{1}\right\|_{2}}\right)
$$

(taking subsequences if necessary to obtain convergence). Moreover $\left\{x_{p}^{2}\right\}$ is bounded and so $d=$ $\lim _{p} \frac{x_{p}^{1}}{\left\|x_{p}^{1}\right\|_{2}} \in \operatorname{ker} A$. By orthogonality $\left\|x_{p}\right\|_{2}^{2}=\left\|x_{p}^{1}\right\|_{2}^{2}+\left\|x_{p}^{2}\right\|_{2}^{2}$ and so the boundedness of $\left\{x_{p}^{2}\right\}$ gives 
$\lim _{p} \frac{x_{p}^{1}}{\left\|x_{p}\right\|_{2}}=\lim _{p} \frac{x_{p}^{1}}{\left\|x_{p}^{1}\right\|_{2}}=d . \quad$ As $x_{p} \in \operatorname{conv} X$ we have $d=\lim _{p} \frac{x_{p}}{\left\|x_{p}\right\|_{2}} \in 0^{+} \operatorname{conv} X$ and so $d \in$ $0^{+} \operatorname{conv}(X) \cap \operatorname{ker} A$. Thus $d:=\lim _{p} \frac{x_{p}^{1}}{\left\|x_{p}^{1}\right\|_{2}} \in \operatorname{ker} A \cap 0^{+} \operatorname{conv}(X)$ and $c d=0$. By construction for all $\varepsilon>0$ there exists $y_{\varepsilon} \in C(\bar{\kappa})$ with $\left\|y_{\varepsilon}-x^{2}\right\|_{2} \leq \varepsilon, c y_{\varepsilon}+\varepsilon \geq c x^{2}$ and $z_{\varepsilon}:=y_{\varepsilon}+\alpha_{\varepsilon} d \in K(\bar{\kappa})$, for some $\alpha_{\varepsilon}>0$. Then we have

$$
\begin{aligned}
\lim _{p} F\left(\kappa_{p}, \lambda\right) & =\lim _{p}\left(c x_{p}^{1}+c x_{p}^{2}+\lambda\left(b-A x_{p}^{2}\right)\right) \leq c x^{2}+\lambda\left(b-A x^{2}\right) \leq c y_{\varepsilon}+\lambda\left(b-A y_{\varepsilon}\right)+\varepsilon\left(1+\|\lambda A\|_{2}\right) \\
& =c z_{\varepsilon}+\lambda\left(b-A z_{\varepsilon}\right)+\varepsilon\left(1+\|\lambda A\|_{2}\right) \leq F(\bar{\kappa}, \lambda)+\varepsilon\left(1+\|\lambda A\|_{2}\right) .
\end{aligned}
$$

As $\varepsilon$ is arbitrary we have $\lim _{p} F\left(\kappa_{p}, \lambda\right) \leq F(\bar{\kappa}, \lambda)$ in both cases, establishing upper semi-continuity.

Consider the mapping

$$
B \mapsto Z(B):=\inf _{\lambda} \max _{\kappa \in[0, B]}[F(\kappa, \lambda)-\sigma \psi(\kappa)] .
$$

which is monotonically non-decreasing and bounded above as $z_{\sigma}^{L D+}<+\infty$. Now as $\kappa \mapsto F(\kappa, \lambda)$ is monotone, it is quasi-concave, its level sets being unbounded intervals. Hence $\kappa \mapsto F(\kappa, \lambda)-\sigma \psi(\kappa)$ is also quasi-concave. It is evident that $\lambda \mapsto F(\kappa, \lambda)$ is convex for fixed $\kappa$. Thus we may apply the Sion minimax theorem. By the previous analysis for every $\varepsilon>0$ there exists $\lambda_{\varepsilon}$ attaining the infimum to within $\varepsilon$ and a $B_{\varepsilon}=B\left(\lambda_{\varepsilon}\right)$ such that

$$
\begin{aligned}
z_{\sigma}^{L D+}+\varepsilon & =Z(+\infty)+\varepsilon>\sup _{\kappa \geq 0}\left[F\left(\kappa, \lambda_{\varepsilon}\right)-\sigma \psi(\kappa)\right]=\max _{\kappa \in\left[0, B_{\varepsilon}\right]}\left[F\left(\kappa, \lambda_{\varepsilon}\right)-\sigma \psi(\kappa)\right] \\
& \geq \max _{\kappa \in\left[0, B_{\varepsilon}\right]} \inf _{\lambda}[F(\kappa, \lambda)-\sigma \psi(\kappa)]=\inf _{\lambda} \max _{\kappa \in\left[0, B_{\varepsilon}\right]}[F(\kappa, \lambda)-\sigma \psi(\kappa)]=Z\left(B_{\varepsilon}\right) \geq Z(0)=z^{I P},
\end{aligned}
$$

where the last equalities follow from an application of the Sion minimax theorem. The maximum in (9) is attained by $\kappa_{\varepsilon}$ due to the upper semi-continuity of $F$ in $\kappa$. Suppose there does not exists a $B>0$ such that $Z(B)=Z(+\infty)$. Then we necessarily have $\left\|\lambda_{\varepsilon}\right\| \rightarrow \infty$ (to assume otherwise results in a bound on $\kappa$ ) and for any $\kappa_{\varepsilon} \in\left[0, B_{\varepsilon}\right]$ attaining the maximum in (9) we must have $\kappa_{\varepsilon} \rightarrow+\infty$ as $\varepsilon \downarrow 0$. Note that (9) implies

$$
z_{\sigma}^{L D+}+\varepsilon \geq \inf _{\lambda} F\left(\kappa_{\varepsilon}, \lambda\right)-\sigma \psi\left(\kappa_{\varepsilon}\right) \geq z^{I P} .
$$

As $\sigma>0$ and $\psi\left(\kappa_{\varepsilon}\right) \rightarrow+\infty$ we have $\inf _{\lambda} F\left(\kappa_{\varepsilon}, \lambda\right) \rightarrow+\infty$ and the following contradiction ensues:

$$
+\infty>z^{L P} \geq z^{L D}=\inf _{\lambda} \sup \{c x+\lambda(b-A x) \mid x \in K(+\infty)\} \geq \lim _{\kappa_{\varepsilon} \rightarrow \infty} \inf _{\lambda} F\left(\kappa_{\varepsilon}, \lambda\right)=+\infty .
$$

Applying the Sion minimax theorem we obtain for a fixed but sufficiently large $B>0$

$$
\begin{aligned}
\inf _{\lambda} \max _{\kappa \geq 0}[F(\kappa, \lambda)-\sigma \psi(\kappa)] & =\inf _{\lambda} \max _{\kappa \in[0, B]}[F(\kappa, \lambda)-\sigma \psi(\kappa)]=\max _{\kappa \in[0, B]} \inf _{\lambda}[F(\kappa, \lambda)-\sigma \psi(\kappa)] \\
& \leq \max _{\kappa \geq 0} \inf _{\lambda}[F(\kappa, \lambda)-\sigma \psi(\kappa)] \leq \inf _{\lambda} \max _{\kappa \geq 0}[F(\kappa, \lambda)-\sigma \psi(\kappa)]
\end{aligned}
$$

forcing equality. Finally (for $B>0$ sufficiently large)

$$
\begin{aligned}
z_{\sigma}^{L D+} & =\inf _{\lambda} z_{\sigma}^{L R+}(\lambda) \\
& =\inf _{\lambda} \max _{\kappa \in[0, B]}[\sup \{c x+\lambda(b-A x) \mid x \in \operatorname{conv}(X \cap\{y \mid\|b-A y\| \leq \kappa\})\}-\sigma \psi(\kappa)] \\
& =\inf _{\lambda} \max _{\kappa \in[0, B]}[F(\kappa, \lambda)-\sigma \psi(\kappa)] \\
& =\max _{\kappa \in[0, B]}\left[\inf _{\lambda} \sup _{x \in X}\{c x+\lambda(b-A x) \mid\|b-A x\| \leq \kappa\}-\sigma \psi(\kappa)\right] \\
& =\max _{\kappa \in[0, B]}[\sup \{c x \mid A x=b, x \in \operatorname{conv}(X \cap\{y \mid\|b-A y\| \leq \kappa\})\}-\sigma \psi(\kappa)]
\end{aligned}
$$

where the last equality comes from the standard argument from the theory of Lagrangian relaxation (see page 328 of [34]). In particular there exists a $\kappa(\sigma) \in[0, B]$ such that

$$
\begin{aligned}
z_{\sigma}^{L D+} & =\sup \{c x \mid A x=b, x \in \operatorname{conv}(X \cap\{y \mid\|b-A y\| \leq \kappa(\sigma)\})\}-\sigma \psi(\kappa(\sigma)) \\
& \leq \sup \{c x \mid A x=b, x \in \operatorname{conv}(X)\}=z^{L D}
\end{aligned}
$$


Now let $G(\kappa):=\sup \{c x \mid A x=b, x \in K(\kappa)\}$ and note that $\kappa \mapsto G(\kappa)$ is a non-decreasing function with $G(\infty)=z^{L D}<+\infty$. As $z_{\sigma}^{L D+}=\max _{\kappa \in[0, B]}[G(\kappa)-\sigma \psi(\kappa)]$ we see that the term $\sigma \psi(\kappa)$ penalizes large values of $\kappa \geq 0$. Suppose the solution $\kappa(\sigma)$ of $\max _{\kappa \in[0, B]}[G(\kappa)-\sigma \psi(\kappa)]$ satisfies $\kappa(\sigma) \geq \delta>0$ for all $\sigma \geq 0$. Then

$$
\begin{aligned}
-\infty & <z^{I P} \leq \lim _{\sigma \rightarrow \infty} z_{\sigma}^{L D+}=\lim _{\sigma \rightarrow \infty} \max _{\kappa \geq 0}[G(\kappa)-\sigma \psi(\kappa)]=\lim _{\sigma \rightarrow \infty} \max _{\kappa \geq \delta}[G(\kappa)-\sigma \psi(\kappa)] \\
& \leq \lim _{\sigma \rightarrow \infty} \max _{\kappa \geq \delta}[G(\infty)-\sigma \psi(\kappa)]=\lim _{\sigma \rightarrow \infty}\left[z^{L D}-\sigma \psi(\delta)\right]=-\infty,
\end{aligned}
$$

which is a contradiction. Thus the solution $\kappa(\sigma)$ tends to zero as $\sigma$ tends to infinity.

More can be said about the behavior of $\kappa(\sigma)$ using the property $\operatorname{diam}_{\operatorname{lev}} \psi \downarrow 0$ as $\delta \downarrow 0$, where $\operatorname{lev}_{\delta} \psi:=\{\kappa \mid \psi(\kappa) \leq \delta\}$. As the value function $\max _{\kappa \in[0, B]}[G(\kappa)-\sigma \psi(\kappa)]$ is monotonically nonincreasing in $\sigma$ and bounded below by $z^{I P}$ we know that the solution $\kappa(\sigma)$ must be contained in a level set

$$
\left\{\kappa \in[0, B] \mid G(\kappa)-\sigma \psi(\kappa) \geq z^{I P}\right\} \quad \text { for all } \quad \sigma \geq \bar{\sigma}>0 .
$$

Note that $G(\cdot)$ is dominated by the following function (as $c z \leq 0$ for all $z \in \operatorname{ker}(A) \cap 0^{+} X^{L P}$ ):

$$
G(\kappa) \leq H(\kappa):=\sup \{c x \mid A x=b, x \in C(\kappa)\} .
$$

By the Berge maximum principle [1] we have $\kappa \mapsto H(\kappa)$ upper semi-continuous. Let $\bar{M}$ be the maximum of $H(\kappa)$ over $\kappa \in[0, B]$. Then by (10) we have

$$
\kappa(\sigma) \in\left\{\kappa \in[0, B] \mid \frac{\bar{M}-z^{I P}}{\sigma} \geq \psi(\kappa)\right\} .
$$

Consequently for any $\delta>0$, there is a $\sigma_{\delta}$ such that for all $\sigma>\sigma_{\delta}, \kappa(\sigma) \leq \operatorname{diam}^{\operatorname{lov}} \delta \psi \leq \delta$.

We also consider the "full" augmented Lagrangian dual $z^{L D *}=\inf _{\sigma \geq 0, \lambda} z_{\sigma}^{L R+}(\lambda)$.

Proposition 3 Posit the assumptions of Theorem 1 then

$$
z^{L D *}=\sup \{c x \mid A x=b, x \in \operatorname{conv}(X \cap\{y \mid\|b-A y\| \leq 0\})\}=z^{I P} .
$$

Proof. From the previous result we have

$$
\begin{aligned}
z^{I P} & \leq z^{L D *}:=\inf _{\sigma \geq 0, \lambda} z_{\sigma}^{L R+}(\lambda) \\
& =\min _{\sigma \geq 0} \inf _{\lambda} z_{\sigma}^{L R+}(\lambda)=\min _{\sigma \geq 0} \max _{\kappa \geq 0}[\sup \{c x \mid A x=b, x \in \operatorname{conv}(X \cap\{y \mid\|b-A y\| \leq \kappa\})\}-\sigma \psi(\kappa)] \\
& \leq \max _{\kappa \geq 0}[\sup \{c x \mid A x=b, x \in \operatorname{conv}(X \cap\{y \mid\|b-A y\| \leq \kappa\})\}-\sigma \psi(\kappa)] \quad \text { for all } \sigma \geq 0 \\
& \rightarrow \sup \{c x \mid A x=b, x \in \operatorname{conv}(X \cap\{y \mid A y=b\})\} \quad \text { as } \sigma \rightarrow \infty \\
& =\sup \{c x \mid x \in \operatorname{conv}(X \cap\{y \mid A y=b\})\}=\sup \{c x \mid x \in X \text { and } A x=b\}=z^{I P} .
\end{aligned}
$$

We now make the observation that when $X$ constrains us to optimize over a finite (but large) set of discrete elements then the parameter $\sigma$ need not go to infinity.

Corollary 1 Suppose that $X$ has a finite number of elements. Then there exist positive constants $\underline{\kappa}$ and $\Delta$ (dependent only on the constraint data in (IP)) such that for all $\sigma \geq \frac{\|c\| \Delta}{\psi(\underline{\kappa})}$, taking $\kappa(\sigma)=0$ will suffice, and hence $z^{I P}=z_{\sigma}^{L D+}$.

Proof. Recall $K(\kappa):=\operatorname{conv}(X \cap\{y \mid\|b-A y\| \leq \kappa\})$ and $K(0)=\operatorname{conv}(X \cap\{y \mid A y=b\})$, the convex hull of the (IP) feasible set. Let $E(\kappa):=\{y \mid A y=b\} \cap K(\kappa)$ and let $\bar{E}:=E(+\infty)=\{y \mid A y=$ $b\} \cap \operatorname{conv}(X)$. Observe that $E(\kappa) \subseteq \bar{E}$ for all $\kappa \geq 0$, and that $\bar{E}$ must be a bounded polyhedron if $X$ is finite. Hence there exists $\Delta>0$ such that the distance between any point in $\bar{E}$ and any point in $K(0)$ $(=E(0))$ is no greater than $\Delta$. Clearly under the condition that $X$ is finite, for all $\kappa$ sufficiently small, say below $\underline{\kappa}>0$, it must be that $K(\kappa)=K(0)$, so $E(\kappa)=E(0)$. Define

$$
\mathcal{K}(\sigma):=\arg \max _{\kappa \geq 0}[\max \{c x \mid x \in E(\kappa)\}-\sigma \psi(\kappa)] .
$$


Let $\sigma>0$ be such that taking $\kappa(\sigma)=0$ will not suffice, i.e. such that $0 \notin \mathcal{K}(\sigma)$. Then for any $\kappa^{*} \in \mathcal{K}(\sigma)$, it must be that $\kappa^{*} \geq \underline{\kappa}$. Let $x^{*}$ be an optimal solution of the (IP) and let $y^{*} \in \arg \max \{c x \mid$ $\left.x \in E\left(\kappa^{*}\right)\right\}$. Since $x^{*} \in K(0)$ and $y^{*} \in \bar{E}$, we have $\left\|y^{*}-x^{*}\right\| \leq \Delta$. Furthermore

$$
c y^{*}-\sigma \psi\left(\kappa^{*}\right)>c x^{*}-\sigma \psi(0) \Rightarrow \sigma \psi\left(\kappa^{*}\right)<c\left(y^{*}-x^{*}\right) \leq\|c\|\left\|y^{*}-x^{*}\right\| \Rightarrow \sigma \psi(\underline{\kappa})<\|c\| \Delta,
$$

since $\psi$ is monotonically increasing, and thus $\sigma<\frac{\|c\| \Delta}{\psi(\underline{\kappa})}$. We conclude that for any $\sigma \geq \frac{\|c\| \Delta}{\psi(\underline{\kappa})}$, it must be that $0 \in \mathcal{K}(\sigma)$, i.e. we may take $\kappa(\sigma)=0$, and the result follows immediately from Theorem 1 .

\section{Comparison with Previous Duality Results}

As noted in the introduction, there now exists a rich theory of duality for very general continuous optimisation problems $[6,7]$. As integrality can be modeled using concave constraints or penalties it is possible to use these results to deduce strong dual for IPs. In this paper, we have allowed the possibility that the optimal solutions of the MIP are not attained; they were not required for Theorem 1 nor for Proposition 3 under assumption 2 part 1). The attainment of solutions appears to be the assumption that has to be made in order to connect the approach we use to those of [6] and [7]. Let us consider the main result of $[6]$. The general problem $(\mathrm{P})$ is defined to be:

$$
v(P):=\max _{x \in X_{0}} \quad f_{0}(x)
$$

where $X_{0}$ is a closed set (that models the constraints) and $f_{0}: \mathbf{R}^{n} \rightarrow \mathbf{R}$ is a continuous function. In [6, Example 3] this set is chosen as $X_{0}:=\left\{x \in \mathbf{R}^{n} \mid \varphi(x)=0\right\}$ and allows the inclusion of concave constraints to enforce the integrality condition. It has been long recognized in IP that continuous optimization over concave constraints can be used to represent the integrality condition. Thus binary problems and all IPs can be modeled within this framework.

Strong duality is established in [6] under the following set of assumptions. We say that $L: \mathbf{R}^{n} \times \Lambda \rightarrow$ $\mathbf{R}$ is a "Lagrangian function" for (11) if it satisfies the following:

$\left(H_{1}\right): f_{0}(x)=L(x, \lambda)$ for all $x \in X_{0}$ and $\lambda \in \Lambda$.

$\left(H_{2}\left(\Lambda_{0}\right)\right)$ : There exists a subset $\Lambda_{0} \subseteq \Lambda$ such that for all $\alpha>v(P)$ and $\delta>0$

$$
\inf _{\lambda \in \Lambda_{0}}\left[\sup _{\substack{x \in X \\ d\left(x, X_{0}\right) \geq \delta}} L(x, \lambda)\right]<\alpha .
$$

$\left(H_{3}\left(\Lambda_{0}\right)\right)$ : For the same subset $\Lambda_{0} \subseteq \Lambda$ we have

$$
f_{0}(x) \geq L(x, \lambda) \quad \text { for all } x \in X, \lambda \in \Lambda_{0} .
$$

$\left(A_{1}\right)$ : For all $\alpha<v(P)$, the level set $\left\{z \in \mathbf{R}^{n} \mid f_{0}(z) \geq \alpha\right\}$ is compact.

Continuing the derivation of $[6]$, define $q(\lambda):=\max _{x \in \mathbf{R}^{n}} L(x, \lambda)$ for a given Lagrangian function $L$. Then the dual (D) problem of $(\mathrm{P})$ is defined to be

$$
v(D):=\min _{\lambda \in \Lambda} q(\lambda)
$$

To apply this to $z^{I P}=\sup \{c x: A x=b, x \in X\}$ where the integrality constraints are in $X$, take $\varphi(x)=(A x-b, d(x, X))$ so that

$$
X_{0}:=\left\{x \in \mathbf{R}^{n} \mid A x=b, d(x, X)=0\right\}=\{x \in X \mid A x=b\},
$$

where $d(x, Y):=\inf \{\|x-y\| \mid y \in Y\}$ for any set $Y \subseteq \mathbf{R}^{n}$.

The condition $\left(A_{1}\right)$ is an unnatural assumption in IP, but if one assumes that the IP attains an optimal solution then one may introduce into $X_{0}$ a box constraint on the variables that is large enough to contain this optimal solution. Then $\left(A_{1}\right)$ may be replaced by an assumption that $X_{0}$ is compact, i.e. for large enough $L$ we have $X_{0}=\left\{x \in X \mid A x=b,-L \leq x_{i} \leq L\right.$, for $\left.i=1, \ldots, n\right\}$. As noted earlier, we did not need assurance of the attainment of a solution to obtain our results in Theorem 1 and 
Proposition 3, however it appears to be needed here in order to apply the result of [6]. Continuing with this assumption one can see that the Lagrangian function

$$
L(x,(\mu, \sigma)):=c x+\mu(b-A x)-\sigma \rho(b-A x)
$$

along with the sets $\Lambda:=\left\{\lambda=(\mu, \sigma) \mid \mu \in \mathbf{R}^{m}\right.$ and $\left.\sigma \geq 0\right\} \quad$ and $\Lambda_{0}:=\{0\} \times \mathbf{R}_{+}$satisfy the assumptions $\left(H_{1}\right)-\left(A_{1}\right)$ as follows.

1. $\left(H_{1}\right)$ : For $x_{0} \in X_{0}, L\left(x_{0},(\mu, \sigma)\right)=c x_{0}=f_{0}\left(x_{0}\right)$.

2. $\left(H_{3}\left(\Lambda_{0}\right)\right)$ : For all $x \in X,(\mu, \sigma) \in \Lambda_{0}$ we have

$$
\begin{aligned}
L(x,(\mu, \sigma)) & :=c x+0(b-A x)-\sigma \rho(b-A x) \\
& =c x-\sigma \rho(b-A x) \leq c x=f_{0}(x)
\end{aligned}
$$

3. $\left(H_{2}\left(\Lambda_{0}\right)\right)$ : This may now be established exactly as in [6, Example 3]. Note that $\left(A_{1}\right)$ is critical for this argument and can be replaced by the assumption of compactness of $X_{0}$.

On application of $[6$, Theorem 2.2] we have $v(P)=v(D)$ or

$$
\begin{aligned}
z^{L D *} & =\inf _{\sigma \geq 0, \lambda} z_{\sigma}^{L R+}(\lambda)=\inf _{\sigma \geq 0, \lambda} \max _{x \in X}[c x+\mu(b-A x)-\sigma \rho(b-A x)] \\
& =\max _{x \in X_{0}} c x=\max \{c x \mid A x \leq b, x \in X\}=z^{I P}
\end{aligned}
$$

where the last equality hold under the assumption that the optimal solution $\bar{x}$ exists and

$$
\bar{x} \in\left\{x \mid-L<x_{i}<L, \text { for } i=1, \ldots, n\right\} .
$$

The intermediate results we have established, in particular, Theorem 1, are not deducible in this way and as a consequence neither is Corollary 1.

\section{Conclusions and Future Work}

Whilst the contribution of this paper is primarily theoretical, we hope that it will stimulate interest in, and provide insights to support, the development of augmented Lagrangian algorithms for IP. One step in that direction may have already occurred, in the form of column generation stabilization techniques [32]: the primal form of the stabilization allows a controlled violation of the constraints relaxed in a corresponding Lagrangian dual problem. However in these settings, the control occurs outside the convexification procedure: it would be interesting to see how this could be moved inside it, to provide a column generation analogue for the augmented Lagrangian dual. A more direct approach may be to approximate the augmented Lagrangian relaxation by use of a "partially diagonalized" penalty term, structured to permit decomposition into smaller independent problems and exploiting the power of MINLP solvers (see, for example, $[4,9,11]$ ) to solve them. Finally we note that recent results of Dey and Moran [12], on properties of convex hulls of integer points in general convex sets, may facilitate extensions of Theorem 1 to the case of pure integer sets by permitting Corollary 1 to be extended to other cases such as the case of an unbounded pure integer set; these will be directions of future research.

\section{Acknowledgements}

We thank two anonymous referees, whose thorough consideration and insightful comments enabled us to substantially improve the paper. We also acknowledge the generous support of the Australian Research Council through Discovery Grant DP0987445, without which this work would not have occurred. 


\section{Appendix}

We will prove the cases of Lemma 1 separately. In all cases the orthogonal decomposition follows from Proposition 1 , so we do not explicitly mention this again. We assume throughout that $z^{L P}<+\infty$. We precede these cases by showing the local uniform boundedness of $\kappa \mapsto C(\kappa)$ for all cases.

\section{Proof. local uniform boundedness for all $0 \leq \kappa<\bar{\kappa}$}

We prove the local uniform boundeness of $\kappa \mapsto P_{V^{\circ}} K(\kappa)$ for the general case using an arbitrary norm $\|\cdot\|$ in the penalty. Denote the Euclidean norm by $\|\cdot\|_{2}$. As the constraint $Q(\kappa):=$ $\{y \mid\|b-A y\| \leq \kappa\}$ only allows $y$ to deviate from $\{x \mid A x=b\}$ by a component in Range $A^{T}$ of maximum norm $\left\|A^{T}\left(A A^{T}\right)^{-1}(A y-b)\right\|_{2} \leq\left\|A^{T}\left(A A^{T}\right)^{-1}\right\|_{2}\|A y-b\|_{2} \leq\left\|A^{T}\left(A A^{T}\right)^{-1}\right\|_{2} \frac{1}{\gamma} \kappa$ (we have invoked the equivalence of norms). Let $\eta:=\frac{1}{\gamma}\left\|A^{T}\left(A A^{T}\right)^{-1}\right\|_{2}$. As $K(\kappa) \subseteq X^{L P} \cap Q(\kappa)$ for all $\bar{\kappa} \geq \kappa \geq 0$ using [35, Corollary 8.3.3], $0^{+} K(\kappa) \subseteq 0^{+} X^{L P} \cap \operatorname{ker} A:=V$ we have

$$
P_{V^{\circ}} K(\kappa) \subseteq P_{V^{\circ}}\left(X^{L P} \cap Q(\bar{\kappa})\right) \subseteq P_{V^{\circ}}\left(X^{L P} \cap Q(0)\right)+\bar{B}_{\eta \bar{\kappa}}(0):=C .
$$

As $X_{0}:=X^{L P} \cap Q(0)$ is a polyhedral convex set with a recession cone $V$ and lineality space $L:=$ $(-V) \cap V$ we may express $X_{0}=\operatorname{conv}\left(\operatorname{ext}\left(X_{0}\right)\right)+V$ where ext denotes the finite set of extremal points of the convex set $X_{0} \cap L^{\perp}$. Thus $P_{V^{\circ}}\left(X^{L P} \cap Q(0)\right) \subseteq P_{V^{\circ}}\left(\operatorname{conv}\left(\operatorname{ext}\left(X_{0}\right)\right)\right.$ which is a bounded set. Hence the right hand side set $C$ in (12) is closed and uniformly bound for all $\bar{\kappa} \geq \kappa \geq 0$ ).

\section{Proof. [Lemma 1: Case 2 of Assumption 2]}

In order to prove this case of Lemma 1, we follow the approach of Meyer [33]. Recall Case 2) of Assumption 2 is that $A$ and $D$ have rational entries and the penalty function uses the $\infty$-norm. Thus we investigate the continuity of $\kappa \mapsto K_{\infty}(\kappa)$ where

$$
\|b-A x\|_{\infty} \leq \kappa \quad \Leftrightarrow \quad x \in Q_{\infty}(\kappa):=\{y \mid b-\kappa \mathbf{1} \leq A y \leq b+\kappa \mathbf{1}\},
$$

for 1 the vector of all ones. In this case, $K_{\infty}^{L P}(\kappa):=X^{L P} \cap Q_{\infty}(\kappa)$ is a polyhedron with rational constraint matrices. By Theorem 2.19 of [10] (see in particular the subsequent Remark 2.20; see also [33]), and since $K_{\infty}(\kappa):=\operatorname{conv}\left(X \cap Q_{\infty}(\kappa)\right) \supseteq K(0)$ is non-empty by our standing assumption that the IP is feasible, it must be that $K_{\infty}(\kappa)$ is a polyhedron with recession cone coinciding with that of $K_{\infty}^{L P}(\kappa)$. Observe that the recession cone of $K_{\infty}^{L P}(\kappa)$ is given by

$$
\begin{aligned}
0^{+} K_{\infty}^{L P}(\kappa) & =0^{+}\left\{x \in X^{L P} \mid b-\kappa \mathbf{1} \leq A x \leq b+\kappa \mathbf{1}\right\} \\
& =\left\{r \in 0^{+} X^{L P} \mid A r=0\right\}=\{r \geq 0 \mid D r=0, A r=0\}=0^{+} X^{L P} \cap \operatorname{ker} A=V
\end{aligned}
$$

independent of $\kappa$. We have thus shown that for all $\kappa \geq 0, K_{\infty}(\kappa)$ is a polyhedron with recession cone precisely $V$.

Suppose initially that we have a pure integer program, i.e. $|J|=n$. Choose a positive constant $M$ such that $\tilde{A}:=M A$ is an integer matrix. Then we have (where $\lfloor\cdot\rfloor$ rounds down and $\lceil\cdot\rceil$ rounds up)

$$
K_{\infty}(\kappa)=\operatorname{conv}\{x \in X \mid\lceil M(b-\kappa \mathbf{1})\rceil \leq \tilde{A} x \leq\lfloor M(b+\kappa \mathbf{1})\rfloor\} .
$$

Considering $\kappa_{q} \downarrow \kappa$, we see that there must exist $\bar{q}$ such that for all $q \geq \bar{q}$ the lower and upper bounds on $\tilde{A} x$ in the definition of $K_{\infty}\left(\kappa_{q}\right)$ are precisely those in the definition of $K_{\infty}(\kappa)$, i.e. $\left\lceil M\left(b-\kappa_{q} \mathbf{1}\right)\right\rceil=$ $\lceil M(b-\kappa \mathbf{1})\rceil$ and $\left\lfloor M\left(b+\kappa_{q} \mathbf{1}\right)\right\rfloor=\lfloor M(b+\kappa \mathbf{1})\rfloor$, and hence $K_{\infty}\left(\kappa_{q}\right)=K_{\infty}(\kappa)$. Continuity follows.

For the mixed integer case we proceed as in [33] Lemma 3.8 amd Theorem 3.9. Without loss of generality suppose the integer variables are those with smallest indices, i.e. $J=\left\{1, \ldots, n_{1}\right\}$, and write $A=\left[\begin{array}{ll}A_{1} & A_{2}\end{array}\right]$ where $A_{1}=A_{J}$ is the matrix formed from columns associated with $J$, and similarly $D=\left[\begin{array}{ll}D_{1} & D_{2}\end{array}\right]$ where $D_{1}=D_{J}$. Observe $K_{\infty}(\kappa)$ has the form

$$
K_{\infty}(\kappa)=\operatorname{conv}\left(\left\{(x, y) \in \mathbf{Z}_{+}^{n_{1}} \times \mathbf{R}^{n-n_{1}} \mid \begin{array}{rl}
-\kappa \mathbf{1} & \leq A_{1} x+A_{2} y \leq b+\kappa \mathbf{1} \\
d & \leq D_{1} x+D_{2} y \leq d \\
0 & \leq y
\end{array}\right.\right.
$$

Making use of the theory of linear programming, we call the pair $(B, r)$ a basis if $B=\left[\begin{array}{ll}B_{1} & B_{2}\end{array}\right]$ is a subset of the rows of

$$
\left[\begin{array}{cc}
A_{1} & A_{2} \\
D_{1} & D_{2} \\
0 & I
\end{array}\right]
$$


chosen so that $B_{2}$ is nonsingular and $r$ is the corresponding subvector of

$$
\left[\begin{array}{c}
b \pm \kappa \mathbf{1} \\
d \\
0
\end{array}\right]
$$

i.e. if the $j$ th row of $B$ corresponds to row $i$ in the original matrix with $i \leq m$, then $r_{j} \in\left\{b_{i}-\kappa, b_{i}+\kappa\right\}$. Let $\mathcal{B}$ denote the set of all such bases. Define $S_{\infty}(\kappa):=\left\{(x, y) \in X \mid b-\kappa \mathbf{1} \leq A_{1} x+A_{2} y \leq b+\kappa \mathbf{1}\right\}$ and for basis $(B, r)$ define $S_{\infty}(\kappa)(B, r):=\left\{(x, y) \mid(x, y) \in S_{\infty}(\kappa), B_{1} x+B_{2} y=r\right\}$. Then we have

$S_{\infty}(\kappa)(B, r)=\left\{(x, y) \in \mathbf{Z}_{+}^{n_{1}} \times \mathbf{R}^{n-n_{1}} \mid B_{1} x+B_{2} y=r, y \geq 0, D_{1} x+D_{2} y=d\right.$ and $\left.b-\kappa \mathbf{1} \leq A_{1} x+A_{2} y \leq b+\kappa \mathbf{1}\right\}$.

Now define the projection onto the integer variables $S_{\infty}(\kappa)(B, r ; J):=\left\{x \in \mathbf{Z}_{+}^{n_{1}} \mid(x, y) \in S_{\infty}(\kappa)(B, r)\right\}$ and observe that

$$
\begin{aligned}
S_{\infty}(\kappa)(B, r ; J)=\left\{x \in \mathbf{Z}_{+}^{n_{1}} \mid\right. & B_{2}^{-1} r-B_{2}^{-1} B_{1} x \geq 0, \quad\left(D_{1}-D_{2} B_{2}^{-1} B_{1}\right) x=d-D_{2} B_{2}^{-1} r \\
& \left.b-\kappa \mathbf{1}-A_{2} B_{2}^{-1} r \leq\left(A_{1}-A_{2} B_{2}^{-1} B_{1}\right) x \leq b+\kappa \mathbf{1}-A_{2} B_{2}^{-1} r\right\} .
\end{aligned}
$$

This is a pure integer feasible set defined by constraints with a rational constraint matrix, $G(B)$, say, independent of $\kappa$ (and $r$ ), and a vector $g(\kappa ; B, r)$ such that $S_{\infty}(\kappa)(B, r ; J)=\left\{x \in \mathbf{Z}_{+}^{n_{1}} \mid G(B) x \leq\right.$ $g(\kappa ; B, r)\}$. By Corollary 3.5 of $[33], S_{\infty}(\kappa)(B, r ; J)$ must have a finite number of extreme points. Observe that $g(\kappa ; B, r)$ has the form

$$
g(\kappa ; B, r)=\left[\begin{array}{c}
B_{2}^{-1} r \\
d-D_{2} B_{2}^{-1} r \\
-d+D_{2} B_{2}^{-1} r \\
b+\kappa \mathbf{1}-A_{2} B_{2}^{-1} r \\
-b+\kappa \mathbf{1}+A_{2} B_{2}^{-1} r
\end{array}\right]
$$

Furthermore there is a positive integer $M$ such that $S_{\infty}(\kappa)(B, r ; J)=\left\{x \in \mathbf{Z}_{+}^{n_{1}} \mid M G(B) x \leq\right.$ $\lfloor M g(\kappa ; B, r)\rfloor\}$. Thus for small $\kappa<\kappa_{B, r}, S_{\infty}(\kappa)(B, r ; J)$ is a constant set independent of $\kappa$; in fact for sufficiently small $\kappa, S_{\infty}(\kappa)(B, r ; J)=S_{\infty}(0)(B, r ; J)$. Hence $\operatorname{conv}\left(S_{\infty}(\kappa)(B, r ; J)\right)$ is a fixed polyhedral set for small $\kappa<\kappa_{B, r}$. Indeed for such small $\kappa$ it must be that $\operatorname{conv}\left(S_{\infty}(\kappa)(B, r ; J)\right)=\mathcal{F}(B):=\{x \mid$ $F(B) x \leq f(B)\}$ for some rational matrix $F(B)$ and rational vector $f(B)$ (by well known results, e.g. in [34], since $M G(B)$ and $\lfloor M g(0 ; B, r)\rfloor$ are integer).

Now we claim that

$$
\operatorname{conv}\left(S_{\infty}(\kappa)(B, r)\right)=\left\{(x, y) \mid x \in \operatorname{conv}\left(S_{\infty}(\kappa)(B, r ; J)\right), y=B_{2}^{-1} r-B_{2}^{-1} B_{1} x\right\}
$$

Containment of $\operatorname{conv}\left(S_{\infty}(\kappa)(B, r)\right)$ in the right-hand side follows easily from first principles. Any extreme point $(x, y)$ of the right-hand side set must by Lemma 3.3 of [33] have $x$ an extreme point of $\operatorname{conv}\left(S_{\infty}(\kappa)(B, r ; J)\right)$, and hence $x \in S_{\infty}(\kappa)(B, r ; J)$ (by Lemma 3.1 of [33]). Denote the finite set of extremal points of $\operatorname{conv}\left(S_{\infty}(\kappa)(B, r ; J)\right)$ by $\mathcal{F}(B)$. It follows (by the definition of $y$ in the right-hand side) that $(x, y) \in S_{\infty}(\kappa)(B, r) \subset \operatorname{conv}\left(S_{\infty}(\kappa)(B, r)\right)$ as required (if the latter set contains all extreme points of the right-hand side set then it's convex hull contains that set).

Thus for $\kappa<\kappa_{B, r}$ it must be that $\operatorname{conv}\left(S_{\infty}(\kappa)(B, r)\right)$ can be represented as a polyhedral set dependent on $\kappa$ only via the continuous variables (recall $r$ may depend on $\kappa$ ):

$$
\operatorname{conv}\left(S_{\infty}(\kappa)(B, r)\right)=\left\{(x, y) \mid x \in \mathcal{F}(B), B_{1} x+B_{2} y=r\right\}
$$

By Theorem 3.9 of [33] we have $K_{\infty}(\kappa)=\operatorname{conv}\left(\bigcup_{(B, r) \in \mathcal{B}} \operatorname{conv}\left(S_{\infty}(\kappa)(B, r)\right)\right)+V$ and as $\mathcal{B}$ has a finite cardinality we deduce that $K_{\infty}(\kappa)$ is a polyhedral set that only depends on $\kappa$ via the continuous variables for $0<\kappa<\min \left\{\kappa_{B, r} \mid(B, r) \in \mathcal{B}\right\}:=\bar{\kappa}$. For each $x$ the system of equations in (14) yields a continuous convex, polyhedral valued multifunction of $\kappa$ (apply the Hoffman's bound [41]). The upper semi-continuity of $K_{\infty}(\kappa)$ follows from the classical facts that finite intersections and finite unions preserves upper semi-continuity as does convex hulls and the addition of $V$.

For the case 1) of Assumption 2 we need another lemma. Denote the complement of a set $R$ by $R^{c}$. In a set which has a pointed recession cone the contribution of integral points to a convex combination must reduce as they become more distant from the point represented. 
Lemma 2 Suppose $K$ is a convex set with $0^{+} K$ a pointed cone. Let $J$ denote a subset of indices $\{1, \ldots, n\}$ to be constrained to integral values in the mixed variable set $I:=\left\{x \in \mathbf{R}^{n} \mid x_{i} \in \mathbf{Z}, i \in J\right\}$. Let $\tilde{c} \in \operatorname{int}\left(0^{+} K\right)^{\circ}$ so that $K \cap R(\alpha)$ is bounded for all $\alpha$, where $R(\alpha):=\{x \mid \tilde{c} x \geq \alpha\}$. Then for all $\varepsilon>0, \alpha \in \mathbf{R}$ there exists fixed numbers $N_{\varepsilon} \in \mathbf{N}, \alpha_{\varepsilon} \in \mathbf{R}$, such that for all $x \in \overline{\operatorname{conv}}(K \cap I) \cap R(\alpha)$ there exists multipliers $\lambda_{i} \geq 0, \sum_{i=1}^{\infty} \lambda_{i}=1$ such that $x=\sum_{i=1}^{\infty} \lambda_{i} x^{i}$, for $x^{i} \in K \cap I$ and for which $\lambda_{\varepsilon}:=\sum_{i=1}^{N_{\varepsilon}} \lambda_{i} \geq 1-\varepsilon, x_{1}:=\sum_{i=1}^{N_{\varepsilon}} \frac{\lambda_{i}}{\lambda_{\varepsilon}} x^{i} \in \operatorname{conv}\left(K \cap R\left(\alpha_{\varepsilon}\right) \cap I\right)$.

Proof. Let $x \in \overline{\operatorname{conv}}(K \cap I) \cap R(\alpha)$ be arbitrary. We first show that $\alpha_{\varepsilon}$ can be chosen so that we must have $\lambda_{\varepsilon} \geq 1-\varepsilon$ whenever $x=\lambda_{\varepsilon} x_{1}+\left(1-\lambda_{\varepsilon}\right) x_{2}$ where $x_{1} \in \operatorname{conv}\left(K \cap R\left(\alpha_{\varepsilon}\right) \cap I\right)$ and $x_{2} \in$ $\overline{\operatorname{conv}}\left(K \cap R\left(\alpha_{\varepsilon}\right)^{c} \cap I\right):=K I\left(\alpha_{\varepsilon}\right)$. This follows easily from the fact that $x$ is constrained to a bounded region and that the recession cone of $K$ is pointed. [We argue by contradiction: if the proposition fails then there exists $\alpha$ and $\varepsilon>0$ and $x^{k} \in \overline{\operatorname{conv}}(K \cap I) \cap R(\alpha)$ with $x^{k}=\lambda^{k} x_{1}^{k}+\left(1-\lambda^{k}\right) x_{2}^{k}$ and $x_{2}^{k} \in K I\left(\alpha^{k}\right)$, $\left\|x_{2}^{k}\right\| \rightarrow+\infty$ as $\left|\alpha^{k}\right| \rightarrow \infty$ with $\varepsilon<1-\lambda^{k}$. Consequently we have $\left(1-\lambda^{k}\right) \frac{x_{2}^{k}}{\left\|x_{2}^{k}\right\|}=\frac{x}{\left\|x_{2}^{k}\right\|}-\lambda^{k} \frac{x_{1}^{k}}{\left\|x_{2}^{k}\right\|}$ for all $k$. Taking convergent subsequences so that $\frac{x_{2}^{k}}{\left\|x_{2}^{k}\right\|} \rightarrow \hat{x}_{2}, \frac{x_{1}^{k}}{\left\|x_{2}^{k}\right\|} \rightarrow \hat{x}_{1}, \lambda^{k} \rightarrow \lambda$ then $0 \leq \lambda<1$ and $(1-\lambda) \hat{x}_{2}=-\lambda \hat{x}_{1}$ with $\left\|\hat{x}_{2}\right\|=1$ (implying $\lambda \neq 0$ ) and so $-\hat{x}_{2}, \hat{x}_{2} \in 0^{+} K$, a contradiction]. Having fixed $\alpha_{\varepsilon}$ we may consider whether there is a fixed number of components in the convex combination required to construct $x_{1}$. Note $K \cap R\left(\alpha_{\varepsilon}\right) \cap I$ contains a finite number of integrally constrained values, i.e. the projection of $K \cap R\left(\alpha_{\varepsilon}\right) \cap I$ onto the variables indexed by $J$ has a finite number of elements, say $M_{\varepsilon}$. It is not hard to show that since $K$ is convex, at most one component having a given integer subvector in this finite set is needed in the convex combination representing $x_{1}$. [To see why, take any convex combination of points in $K \cap R\left(\alpha_{\varepsilon}\right) \cap I$ yielding $x_{1}$, and gather together the terms for each distinct integer subvector. Since $K \cap R(\alpha)$ is convex, the terms with the same integer subvector must be a convex combination of points in a convex set and hence can be represented by a single point in this set.] Thus we may take $N_{\varepsilon}:=M_{\varepsilon}$.

Proof. [Lemma 1: Cases 1 and 3 of Assumption 2] Now assume that Case 1) of Assumption 2 holds, i.e. that the LP relaxation does not have a lineality space in its solution set. Note that $0^{+} K(\kappa) \subseteq$ $0^{+} X^{L P} \cap \operatorname{ker} A=V$, a pointed cone. Let $\tilde{c} \in \operatorname{int}\left(V^{\circ}\right)$ so that for all $\alpha$ the region $R(\alpha) \cap X^{L P} \cap Q(\bar{\kappa})$ is convex and bounded, where $\{x \mid \tilde{c} x \geq \alpha\}:=R(\alpha)$. Because $K\left(\kappa^{\prime}\right) \subseteq K(\kappa)$ for all $\kappa^{\prime} \leq \kappa$, to study graph closure of $\kappa \mapsto \overline{C(\kappa)}$ we need to establish for all $\alpha$ that $\lim \sup _{q} K\left(\kappa_{q}\right) \cap R(\alpha) \subseteq \bar{K}(\kappa)$ for $\kappa_{q} \downarrow \kappa$. If this is so then $\left\{K\left(\kappa_{q}\right)\right\}$ converges to $K(\kappa)$ (see [37, Theorem 4.10]). It suffices to consider $x_{q} \in K\left(\kappa_{q}\right) \cap R(\alpha)$ for a fixed $\alpha$ with $x_{q} \rightarrow x \in R(\alpha) \cap X^{L P} \cap Q(\bar{\kappa})$. Denoting $y_{q}=P_{V^{\circ}}\left(x_{q}\right)$ we then we wish to establish that $\lim _{q} y_{q}:=y \in \overline{C(\kappa)}$.

Now consider $\kappa_{q} \downarrow \kappa$. As $x_{q} \in K\left(\kappa_{q}\right)$ applying Lemma 2, for all $\varepsilon>0$ there exists $\alpha_{\varepsilon}$ and $N_{\varepsilon}$ with the following properties. We have $x_{q}=\sum_{i} \lambda_{i}^{q} x_{q}^{i}$ for $x_{q}^{i} \in X$ with $\sum_{i} \lambda_{i}^{q}=1, \lambda_{q}^{i} \geq 0$ and $\left\|b-A x_{q}^{i}\right\| \leq \kappa_{q}$. Also $\lambda^{q}:=\sum_{i=1}^{N_{\varepsilon}} \lambda_{i}^{q} \geq 1-\varepsilon, x_{1}^{q}:=\sum_{i=1}^{N_{\varepsilon}} \frac{\lambda_{i}^{q}}{\lambda^{q}} x_{q}^{i}, x_{2}^{q}:=\sum_{i>N_{\varepsilon}} \frac{\lambda_{i}^{q}}{1-\lambda^{q}} x_{q}^{i}$ then $x_{q}=\lambda^{q} x_{1}^{q}+\left(1-\lambda^{q}\right) x_{2}^{q} \rightarrow x$ and

$$
x_{1}^{q} \in \operatorname{conv}\left(X \cap R\left(\alpha_{\varepsilon}\right) \cap Q\left(\kappa_{q}\right) .\right.
$$

Taking successive subsequences we may also (after a renumbering of these subsequences) assume $x_{q}^{i} \rightarrow$ $x^{i}$ for all $i=1, \ldots, N_{\varepsilon}$. As $y_{q}, P_{V^{\circ}} x_{2}^{q}, P_{V^{\circ}} x_{1}^{q} \in C$ are uniformly bounded we may take further subsequences (and after renumbering) assume $y_{q} \rightarrow y, \lim _{q} x_{1}^{q}=x_{1}$ and as $\lambda^{q} \geq 1-\varepsilon$ we have $y_{q}=\lambda^{q} P_{V^{\circ}} x_{1}^{q}+\left(1-\lambda^{q}\right) P_{V^{\circ}} x_{2}^{q} \rightarrow y \in P_{V^{\circ}} x_{1}+B_{\varepsilon 2 M}(0)$. By the finiteness of $N_{\varepsilon}$ there exists $\varepsilon>0$ such that $K(\kappa+\varepsilon) \cap R\left(\alpha_{\varepsilon}\right)$ contains no new integral points in the sense that if the $j$ th component $\left(x_{q}^{i}\right)_{j} \in \mathbf{Z}$ is part of the integrality constraint we have $\left(x_{q}^{i}\right)_{j}=\left(x^{i}\right)_{j}$ for $\kappa_{q}<\kappa+\varepsilon$. On fixing these components to their limiting values and denoting by $A_{n-J}$ the matrix with derived from $A$ by deleting all columns in $A_{J}$ associated with the integrally constrained components (indexed by $J$ ) and $b^{i}:=b-\left(A_{J}\left(x^{i}\right)_{J}\right)$, where $\left(x_{q}^{i}\right)_{J}$ are the fixed integrally constrained components $\left(x^{i}\right)_{J}$. We then have all the continuous variable constrained to satisfy $\left\|b^{i}-A_{n-J}\left(x_{q}^{i}\right)_{n-J}\right\| \leq \kappa_{q}$ for all $i=1, \ldots, N_{\varepsilon}$, where $\left(x_{q}^{i}\right)_{n-J}$ denotes the vector obtained from $x_{q}^{i}$ by deleting all the components indexed in $J$. Denote by $g^{i}(y):=\left\|b^{i}-A_{n-J} y\right\|$ and $S^{i}(\kappa):=\left\{y \mid g^{i}(y) \leq \kappa\right.$ and $\left.\left(\left(x^{i}\right)_{J}, y\right) \in X \cap R\left(\alpha_{\varepsilon}\right)\right\}$. Then the Hoffman bound [41] supplies a constant $r^{i}>0$ such that $d\left(\left(x_{q}^{i}\right)_{n-J}, S^{i}(\kappa)\right) \leq r^{i}\left[g^{i}\left(\left(x_{q}^{i}\right)_{n-J}\right)-\kappa\right]_{+} \leq r^{i}\left[\kappa_{q}-\kappa\right]$. Thus in the limit we have $\kappa_{q} \downarrow \kappa$ and $\left(x^{i}\right)_{n-J} \in S^{i}(\kappa)$. Hence for all $i=1, \ldots, N_{\varepsilon}$ we have $\left\|b-A x^{i}\right\| \leq \kappa$ and $x_{1} \in \operatorname{conv}\left[K(\kappa) \cap R\left(\alpha_{\varepsilon}\right) \cap Q(\kappa)\right]$ and so $y \in P_{V^{\circ}} K(\kappa)+B_{\varepsilon \eta}(0)$. As $\varepsilon>0$ was arbitrary we have $y \in \overline{P_{V^{\circ}} K(\kappa)}=\overline{C(\kappa)}$. The uniform boundedness and the closed graph property of $\kappa \mapsto \overline{C(\kappa)}$ allows 
the application of [1, Proposition 6.3.2] to obtain the upper semi-continuity result, thus completing our proof under Case 1) of Assumption 2.

The above arguments follows through in a simplified form when we assume $\operatorname{conv}(X)$ is bounded and hence contains a finite number of integrally constrained values. This establishes the result under Case 3) of Assumption 2.

\section{References}

[1] G. Beer (1993). Topologies on Closed and Closed Convex Sets, Mathematics and its Applications Vol. 268, Kluwer Acad. Publ.

[2] P. Benchimol, G. Desaulniers and J. Desrosiers, "Stabilized dynamic constraint aggregation for solving set partitioning problems", Euro. J. Operational Research (2012), to appear.

[3] C.E. Blair and R. Jeroslow, "The value function in mixed integer programming 1", Discrete Mathematics, (1977) 19:121-138.

[4] P. Bonami, L.T. Biegler, A.R. Conn, G. Cornujols, I.E. Grossmann, C.D. Laird, J. Lee, A. Lodi, F. Margot, N. Sawaya and A. Wächter, "An algorithmic framework for convex mixed integer nonlinear programs", Discrete Optimization, (2008) 5(2):186204.

[5] S. Boyd, N. Parikh, E. Chu, B. Peleato and J. Eckstein, "Distributed optimization and statistical learning via the alternating direction method of multipliers", Foundations and Trends in Machine Learning, (2010) 3:1-122.

[6] R. Burachik and A. Rubinov, "On the absence of duality gap for Lagrangian-type functions", Journal of Industrial and Management Optimisation, (2005) 1:33-38.

[7] R. Burachik and A. Rubinov, "Abstract convexity and augmented Lagrangians", SIAM J. Opt., (2007) 18:413-436.

[8] R. Burachik and Y.C. Kaya, "A deflected subgradient algorithm using a general augumented Lagrangian duality with implications on penalty methods", In: R.S. Burachik and J-C Yao (eds.), Variational Analysis and Generalized Differentiation in Optimization and Control, Springer Optim. Appl., 47, Springer, New York, (2010) 109-132.

[9] M.R. Bussieck and S. Vigerske, "MINLP solver software", In: J.J. Cochran, L.A. Cox, P. Keskinocak, J.P. Kharoufeh, and J. Cole Smith, (eds.), Wiley Encyclopedia of Operations Research and Management Science, 2010.

[10] M. Conforti, G. Cornuéjols and G. Zambelli, "Polyhedral approaches to mixed integer linear programming", In: M. Jünger, T.M. Liebling, D. Naddef, G.L. Nemhauser, W.R. Pulleyblank, G. Reinelt, G. Rinaldi and L.A. Wolsey (eds.), 50 Years of Integer Programming: 1958-2008, Springer, New York, (2010) 343-385.

[11] C. DAmbrosio and A. Lodi, "Mixed integer nonlinear programming tools: a practical overview" 4OR, (2011) 9:329349.

[12] S.S. Dey and D.A. Moran R., "Some properties of convex hulls of integer points contained in general convex sets", Math. Progr., (2013) 141:507-526.

[13] G. Desaulniers, J. Desrosiers and M.M. Solomon, Column Generation, Springer, 2005.

[14] J. Eckstein and D.P. Bertsekas, "On the Douglas-Rachford splitting method and the proximal point algorithm for maximal monotone operators", Math. Progr., (1992) 55:293-318.

[15] I. Elhallaoui, A. Metrane, F. Soumis and G. Desaulniers, "Multi-phase dynamic constraint aggregation for set partitioning type problems", Math. Progr., (2010) 123(2):345-370.

[16] R. D. Galvão, L. G. A. Espejo and B. Boffey, "A comparison of Lagrangian and surrogate relaxations for the maximal covering location problem", European Journal of Operational Research, (2000) 124:377-389. 
[17] A.M. Geoffrion, "Lagrangian relaxation for integer programming", Math. Progr. Studies, (1974) $2: 82-114$.

[18] F. Glover, "A multiphase-dual algorithm for the zero-one integer programming problem", Operations Research, 1965 13:879-919.

[19] F. Glover, "Surrogate constraint duality in mathematical programming", Operations Research, 1975 23:434-451.

[20] R. Gomory, "Some polyhedra related to combinatorial problems", Linear Algebra and Applications (1969) 2:451-558.

[21] H. J. Greenberg and W. P. Pierskalla, "Surrogate mathematical programming", Operations Research, 1970 18:924-939.

[22] J.N. Hooker, "Inference duality as a basis for sensitivity analysis", in E.C. Freudern (ed.), Principles and Practice of Constraint Programming-CP96, LNCS Vol. 1118, Springer, 1996, pp. 224-236.

[23] E. Johnson, "Cyclic groups, cutting planes and shortest path", in: T. Hu and S.Robinson (Eds.), Mathematical Programming, Academic Press, New York, 1973, pp. 185-211.

[24] E. Johnson, Integer Programming: Facets, Subadditivity, and Duality for Group and Semi-group Problems, Society for Industrial and Applied Mathematics, 1980.

[25] M. Karwan and R. Rardin, "Surrogate dual multiplier search procedures in integer programming", Operations Research, (1984) 32(1):52-69.

[26] M. Karwan and R. Rardin, "Some relationships between Lagrangian and surrogate duality in integer programming", Mathematical Programming, (1979), 17(1):320-334.

[27] D. Klabjan, "Subadditive approaches in integer programming", Euro. J. Oper. Res., (2007) 183:525-545.

[28] J. B. Lasserre, "Generating functions and duality for integer programs", Discrete Optimisation, (2005) 2(1):167-187.

[29] J.B. Lasserre, "Integer programming, duality and superadditive functions", Contemporary Mathematics, (2005) 374:139-150.

[30] D. Li and X. Sun, Nonlinear Integer Programming, International Series in Operations Resarch \& Management, Springer, 2006.

[31] D. Li, J. Wang and X. L. Sun, "Computing exact solution to nonlinear integer programming: convergent Lagrangian and objective level cut method", Journal of Global Optimization, (2007) 39:127-154.

[32] O. du Merle, D. Villeneuve, J. Desrosiers and P. Hansen, "Stabilized column generation", Discrete Mathematics, (1999) 194(1-3):229-237.

[33] R.R. Meyer, "On the existence of optimal solutions to integer and mixed integer problems", Math. Progr. (1974) 7:223-235.

[34] G. L. Nemhauser and L. A. Wolsey, Integer and Combinatorial Optimization, Wiley-Interscience, 1988.

[35] R.T. Rockafellar, Convex Analysis, Princeton University Press, 1970.

[36] R.T. Rockafellar, "Augmented Lagrangians and applications of the proximal point algorithm in convex optimization", Math. Oper. Res., (1976) 1:97-116.

[37] Rockafellar, R. T. and Wets R J-B. Variational Analysis, A series of comprehensive studies in mathematics, 317, Springer.

[38] M. Sinclair, "Augmented Lagrangean relaxations in general mixed integer programming", Quaestiones Math., (1978/79) 3:115-146. 
[39] M. Sion, "On general minimax theorems", Pac. J. Math., (1958) 8:171-176.

[40] L. A. Wolsey, "Integer programming duality: price functions and sensitivity analysis", Mathematical Programming, (1981) 20:173-195.

[41] C. Zalinescu, "A nonlinear extension of Hoffman's error bound for linear inequalities", Mathematics of Operations Research, (2003) 28:524-532. 


\section{University Library}

\section{- M M I E R R V A gateway to Melbourne's research publications}

Minerva Access is the Institutional Repository of The University of Melbourne

Author/s:

Boland, NL;Eberhard, AC

Title:

On the augmented Lagrangian dual for integer programming

Date:

2015-05-01

Citation:

Boland, N. L. \& Eberhard, A. C. (2015). On the augmented Lagrangian dual for integer programming. MATHEMATICAL PROGRAMMING, 150 (2), pp.491-509. https:// doi.org/10.1007/s10107-014-0763-3.

Persistent Link:

http://hdl.handle.net/11343/282849 